\title{
Optimal storage and recall with biologically plausible synapses
}

Cristina Savin, Máté Lengyel

Computational \& Biological Learning Lab, Department of Engineering, University of Cambridge

Synaptic plasticity is widely accepted to underlie learning and memory. Yet, models of associative networks with biologically plausible synapses fail to match brain performance: memories stored in such networks are quickly overwritten by ongoing plasticity (Amit \& Fusi 1996, Fusi et al 2007). Metaplasticity - the process by which neural activity changes the ability of synapses to exhibit further plasticity - is believed to increase memory capacity (Fusi et al 2005). However, it remains unclear if neurons can make use of this additional information during recall. In particular, previous attempts at reading out information in metaplastic synapses using heuristic recall dynamics led to rather poor performance (Huang \& Amit 2010).

Here, we developed a theoretical framework for storage and recall with finite-state synapses that allowed us to find neural and synaptic dynamics that maximize the efficiency of autoassociative recall. Since information storage by synaptic plasticity is lossy, we formulated the problem of recalling a previously stored pattern from a noisy cue as probabilistic inference (Lengyel et al 2005) and derived neural dynamics efficiently implementing such inferences. Our approach is general and can be applied to any synaptic plasticity model which involves stochastic transitions between a finite set of states. We show how synaptic plasticity rules need to be matched to the statistics of stored patterns, and how recall dynamics need to be matched both to input statistics and to the plasticity rule itself in order to achieve optimal performance. In particular, for binary synapses with metastates we demonstrate for the first time that memories can be efficiently read out with biologically plausible network dynamics that we derive directly from the synaptic metaplasticity rule with virtually no free parameters.
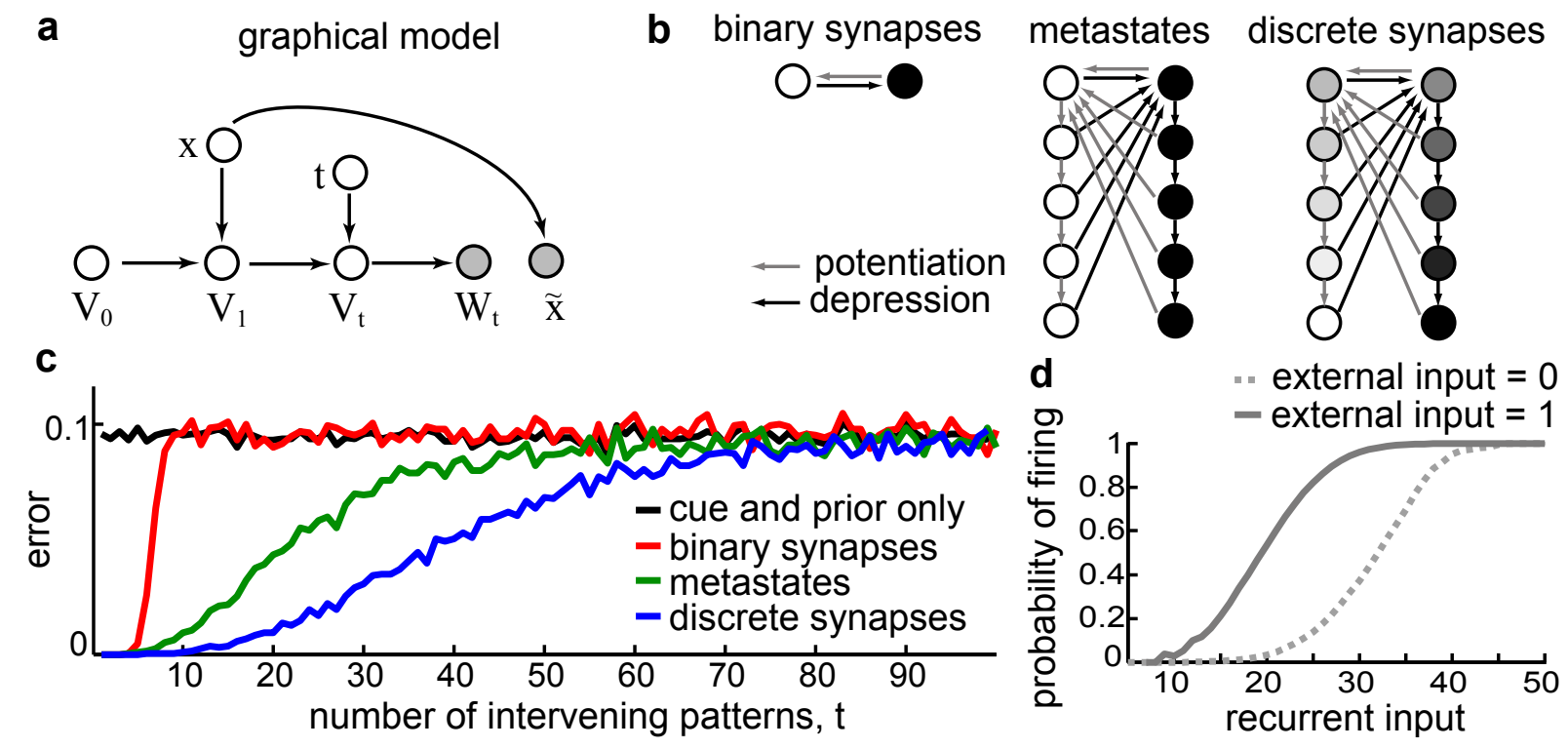

Learning involves stochastic transitions between synaptic metastates $\mathbf{V}$. A pattern $\mathbf{x}$ is recalled given a recall cue, $\tilde{\mathbf{x}}$, and the "visible" efficacies of synapses, $\mathbf{W}$, after a sequence of $t$ other patterns have been stored. The effects of storing multiple patterns could be modeled as a Markov process in synaptic weight space. Assuming a prior over $t$, stochastic recall dynamics were derived as implementing Gibbs sampling from the posterior distribution, $\mathrm{P}(\mathbf{x} \mid \tilde{\mathbf{x}}, \mathbf{W})$, that expresses the probability of any activity pattern $\mathbf{x}$ being the correct response for the recall query given a noisy recall cue, $\tilde{\mathbf{x}}$, and the synaptic efficacies in the network, $\mathbf{W}(a)$. We show that recall from binary synapses with metastates (Fusi et al 2005) is substantially better than from simple binary synapses, and it is nearly as efficient as when all synaptic states have distinct efficacies $(b, c)$. Moreover, optimal recall in this case was well approximated by a sigmoid function of the total input received through recurrent connections, with an additive effect from external input $(d)$. Finally, the amount of information stored in synapses was estimated as the mutual (Shannon) information between the encoded pattern, $\mathbf{x}$, and the synaptic efficacy, $\mathbf{W}_{t}$, which we found to decay either exponentially (binary synapses), or as a power law (binary synapses with metastates). Optimal learning parameters were determined by maximizing the average $\mathrm{MI}\left(\mathbf{x}, \mathbf{W}_{t}\right)$ numerically. 